\title{
Specialized Smooth Muscle Cell Progenitors in Pulmonary Hypertension
}

\author{
Fatima Zahra Saddouk, Aglaia Ntokou, and Daniel M. Greif
}

\begin{abstract}
The accumulation of excessive and ectopic smooth muscle cells (SMCs) is integral to the pathogenesis of diverse cardiovascular diseases, including atherosclerosis, restenosis and pulmonary hypertension. Unfortunately, underlying mechanisms are poorly understood which markedly limits therapeutic options. In the idiopathic form of pulmonary hypertension, reduced pulmonary artery compliance is a strong independent predictor of mortality (Mahapatra et al. J Am Coll Cardiol 47(4):799-803, 2006). Distal arteriole endothelial tubes in the normal lung lack a SMC coating, and pathological distal arteriole muscularization contributes to the reduced compliance. Recently, we identified specialized progenitors that are located at the muscular-unmuscular border of each arteriole in the normal lung and express markers of SMCs and the undifferentiated mesenchyme marker platelet-derived growth factor receptor- $\beta$ (Sheikh et al. Cell Rep 6(5):809-17, 2014; Sci Transl Med 7(308):308ra159, 2015). Upon exposing mice to hypoxia, these "primed" SMC progenitors are induced to express the pluripotency factor Kruppel-like factor 4 (Sheikh et al. Sci Transl Med 7(308):308ra159, 2015). Subsequently, one of these primed cells migrates distally and in a cell autonomous hypoxia-inducible factor (HIF)1- $\alpha$-dependent manner and clonally expands, giving rise to distal arteriole SMCs (Sheikh et al. Sci Transl Med 7(308):308ra159, 2015; Cell Rep 23(4):1152-65, 2018). Additionally, endothelial cell HIF1- $\alpha$-dependent signaling contributes in a noncell autonomous manner, regulating induction, proliferation and differentiation of primed cells (Sheikh et al. Cell Rep 23(4):1152-65, 2018).
\end{abstract}

F. Z. Saddouk · A. Ntokou · D. M. Greif $(\bowtie)$

Section of Cardiovascular Medicine, Department of Internal Medicine, Yale Cardiovascular Research Center, Yale University School of Medicine, New Haven, CT, USA

Department of Genetics, Yale University School of Medicine, New Haven, CT, USA

e-mail: daniel.greif@yale.edu

(C) The Editor(s) (if applicable) and The Author(s) 2020

T. Nakanishi et al. (eds.), Molecular Mechanism of Congenital Heart Disease

and Pulmonary Hypertension, https://doi.org/10.1007/978-981-15-1185-1_4 


\section{Keywords}

Smooth muscle cells $\cdot$ Pulmonary hypertension $\cdot$ Progenitors $\cdot$ Clonality $\cdot$ Vascular

\subsection{Introduction}

Physiologically, pulmonary hypertension $(\mathrm{PH})$ is defined by a mean pulmonary artery pressure greater than or equal to $25 \mathrm{~mm} \mathrm{Hg}$ at rest. PH encompasses heterogeneous entities that the World Health Organization has classified into five groups by etiology as follows: Group 1-pulmonary arterial hypertension (PAH); Group $2-\mathrm{PH}$ due to left heart disease; Group 3- $\mathrm{PH}$ due to lung disease and/or chronic hypoxia; Group 4-PH due to chronic thromboembolism; and Group 5-PH with unclear multi-factorial mechanisms [1]. Overall, $\mathrm{PH}$ is a grave complication of other pathologies as well as a devastating disease in its own right: indeed, within seven years of initial diagnosis of PAH, one-half of patients have died [2].

Histopathological changes in PH include an increased SMC burden in the pulmonary vasculature with muscularization of normally non-muscularized distal pulmonary arterioles. Reduced compliance of the pulmonary arterial vasculature is an independent predictor of mortality in idiopathic PAH [3], and the distal extension of SMC coverage to normally unmuscularized distal arterioles contributes to this reduced arterial compliance. Treatments for PAH are largely limited to therapies that primarily induce vascular dilation, but these approaches do not ameliorate the hypermuscularization. The lack of therapeutic options that combat hypermuscularization is striking but perhaps not surprising given our limited understanding of the molecular and cellular processes underlying distal arteriole muscularization. To begin to delineate these mechanisms, our group utilized a reductionist approach and investigated in-depth, the process of hypoxia-induced distal muscularization of select arteriole beds in the murine lung. We identified novel SMC progenitors located at the muscular-unmuscular arteriole border that have a unique molecular signature and are the source of the vast majority of pathological distal arteriole SMCs in PH. During the muscularization process, these progenitors and their daughter cells undergo a stereotyped program of gene expression, migration and proliferation, which is subjected to intricate cell autonomous and non-cell autonomous regulation.

\subsection{Hypoxia-Induced Distal Pulmonary Arteriole SMCs Derive from Specialized SMC Progenitors}

Chronic hypoxia is a common cause of PH in humans and is widely used in a rodent model of $\mathrm{PH}$, resulting in muscularization of distal pulmonary arterioles $[4,5]$. At each arteriole muscular-unmuscular transition zone in the normal lung, we identified a pool of cells that express SMC markers ( $\alpha$-smooth muscle actin [SMA], smooth muscle myosin heavy chain [SMMHC]) and the undifferentiated mesenchyme marker platelet-derived growth factor receptor (PDGFR- $\beta$ ) [6, 7]. Because 
of their gene expression and position, we assessed if these cells give rise to distal pulmonary arteriole SMCs during $\mathrm{PH}$ through fate mapping in mice carrying the ROSA26R-mTmG Cre reporter and either Acta2-CreER ${ }^{T 2}$ to induce recombination in SMCs (Acta2 is the gene encoding SMA) or Pdgfrb-CreER ${ }^{T 2}$ [6-9]. Treatment of these mice with hypoxia revealed that more than $80 \%$ of the distal pulmonary arteriole SMCs derive from primed SMCs [6, 7]. Thus, we termed these PDGFR$\beta^{+} \mathrm{SMA}^{+} \mathrm{SMMHC}^{+}$progenitors as "primed" cells by virtue of their head start on other SMCs with regard to muscularizing the distal arteriole due to their location and PDGFR- $\beta$ expression.

\subsection{Stereotyped Program of Distal Muscularization}

During the distal muscularization process, the primed SMC-derived lineage undergoes stereotyped steps of dedifferentiation (downregulation of SMMHC and maintenance of PDGFR- $\beta$ expression) and migration beyond the muscularizedunmuscularized arteriole border and finally differentiation (upregulation of SMMHC and downregulation of PDGFR- $\beta$ ) [6]. In addition, analysis of bromodeoxyuridine (BrdU) incorporation indicates that proliferation of distal arteriole SMCs peaks at day 7 of hypoxia with remarkably $2 / 3$ of distal arteriole SMCs staining for BrdU, four hours after a single BrdU injection (100 mg/kg) [6].

\subsection{Monoclonal Expansion of SMCs in PH}

Furthermore, we analyzed the clonal relationship of hypoxia-induced distal arteriole SMCs. For these studies we used Acta2-CreER ${ }^{T 2}$ mice also carrying the multicolor Rainbow $(\mathrm{Rb})$ Cre reporter; a $\operatorname{ROSA26R}(R b /+)$ cell that has undergone Cre-induced recombination will randomly and permanently express a specific fluorophore-Cerulean, mCherry or mOrange-and all of the cell's progeny will express this same fluorophore [10, 11]. Remarkably, for each distal arteriole, almost all of the pathological distal SMCs are marked by a single color (Fig. 4.1) and thus derive from clonal expansion of a single $\mathrm{SMA}^{+}$cell and based on the fate mapping studies described above, from a single primed SMC [6, 7].

\subsection{Signaling Pathways Regulating Primed Cells}

The expression of the pluripotency factor Kruppel-like factor 4 (KLF4) in primed cells is essential for their initial migration in response to hypoxia, but KLF4 is dispensable for subsequent steps of SMC proliferation [7]. Interestingly, the hypermuscularized arterioles of human PH patients have a marked upregulation of KLF4 [7]. Platelet-derived growth factor (PDGF)-B is integral in primed cell KLF4 expression and distal muscularization as $P d g f b(+/-)$ mice are protected against these hypoxiainduced changes and do not develop $\mathrm{PH}[7]$. 


\section{Acta2-CreER, ROSA26R(Rb/+)}

a

Hypoxia

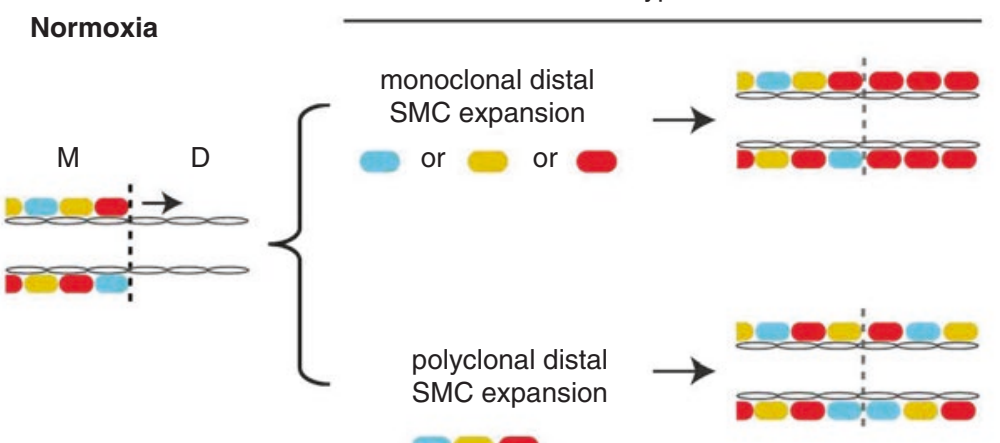

b

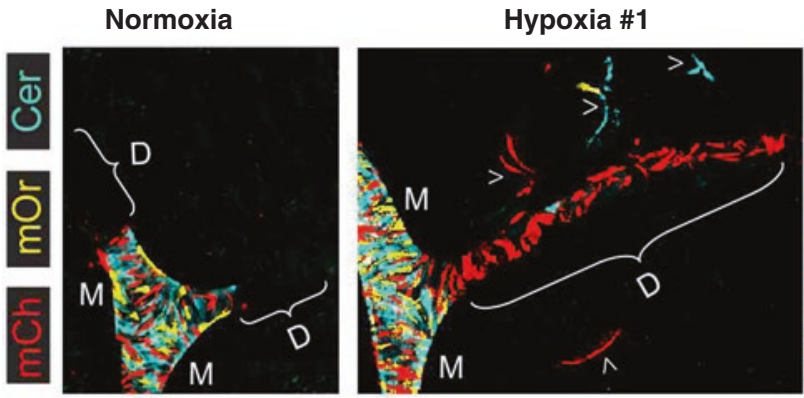

Hypoxia \#2

C

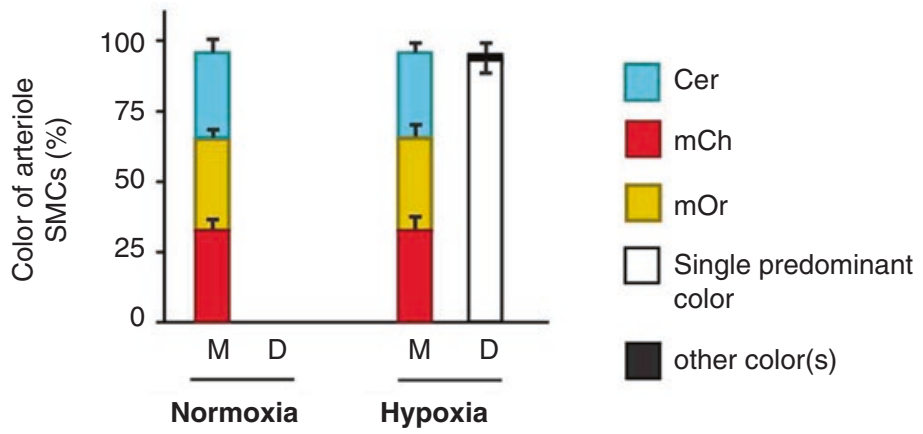

Fig. 4.1 The vast majority of hypoxia-induced SMCs in each pulmonary arteriole derive from a single pre-existing SMC. Acta2-CreER mice also carrying the multi-color Rainbow $(\mathrm{Rb}) \mathrm{Cre}$ reporter $R O S A 26 R(R b /+)$ were induced with tamoxifen, rested and then exposed to normoxia or hypoxia $\left(\mathrm{FiO}_{2} 10 \%\right)$ for 21 days. (a) Potential patterns of Rb colors in pulmonary arteriole SMCs. Middle $(M)$ arteriole SMCs are present in normoxia and marked by different Rb colors [i.e., Cerulean (blue), mOrange (yellow) or mCherry (red)]. Hypoxia-induced distal $(D)$ arteriole SMCs will be a single color if they derive from a single pre-existing SMC. Instead, distal arteriole SMCs will be of multiple colors if they derive from multiple SMCs. (b), Lung sections from a normoxic mouse and two hypoxic mice and imaged for Rb colors. Middle arteriole SMCs are polyclonal whereas distal arterioles are almost entirely monoclonal. Alveolar myofibroblasts are also marked (arrowheads). (c) Quantification of arteriole SMCs by size $(M, D)$ and color. $n=4$ lungs, $2-3$ arterioles per lung (Adapted from reference [7]) 
Hypoxia-inducible factors (HIFs) are key transcription factors in PH. Deletion of Hifla in $\mathrm{SMMHC}^{+}$cells attenuates hypoxia-induced pulmonary vascular remodeling [12]. In contrast to KLF4, our studies suggest that HIF1- $\alpha$ in PDGFR- $\beta^{+}$cells is not required for initial primed cell migration but is needed for the subsequent clonal expansion. In ECs, HIF1- $\alpha$ is upstream of PDGF-B, and EC deletion of either of these genes non-cell autonomously attenuates hypoxia-induced primed cell KLF4 expression, distal muscularization and $\mathrm{PH}[7,13]$.

\subsection{Future Direction and Clinical Implications}

A number of questions are raised by these findings. From a developmental perspective, it is interesting to consider what is the role of primed cells in morphogenesis of the pulmonary vasculature and when do primed cells form. It remains to be determined whether the primed SMCs clonally expand in models of PH other than the hypoxia model and whether primed cells are present in the pulmonary arterioles of other animals beyond mice and in humans. Yet, the clonal expansion of SMC marker ${ }^{+}$progenitors in pathological settings is not limited to hypoxia-induced $\mathrm{PH}$ as a series of recent studies demonstrate marked expansion of rare SMCs during atherogenesis in mice [14-16]. The need for effective and safe treatments for PH is dire, and further studies that investigate the role of primed cells and underlying signaling pathways in pathogenesis are critical and likely to provide key steps in the development of novel therapeutic approaches.

Acknowledgments F.Z.S was supported by Yale University (Brown-Coxe fellowship). This work was funded by the March of Dimes (Basil O'Connor Award, 5-FY13-208 and Gene Discovery \& Translational Grant, 6-FY15-223 to D.M.G.), American Lung Association (Biomedical Research Grant, RG-310716 to D.M.G.), American Heart Association (National Innovative Research Grant, 15IRG23150002 to D.M.G.) and National Institutes of Health (R01HL125815, R01HL133016 and R01HL142674 to D.M.G. and CTSA 5UL1RR024139-08, National Center for Research Resources).

\section{References}

1. Simonneau G, Gatzoulis MA, Adatia I, Celermajer D, Denton C, Ghofrani A, et al. Updated clinical classification of pulmonary hypertension. J Am Coll Cardiol. 2013;62(25 Suppl):D34-41.

2. Benza RL, Miller DP, Barst RJ, Badesch DB, Frost AE, McGoon MD. An evaluation of longterm survival from time of diagnosis in pulmonary arterial hypertension from the REVEAL Registry. Chest. 2012;142(2):448-56.

3. Mahapatra S, Nishimura RA, Sorajja P, Cha S, McGoon MD. Relationship of pulmonary arterial capacitance and mortality in idiopathic pulmonary arterial hypertension. J Am Coll Cardiol. 2006;47(4):799-803.

4. Stenmark KR, Fagan KA, Frid MG. Hypoxia-induced pulmonary vascular remodeling: cellular and molecular mechanisms. Circ Res. 2006;99(7):675-91.

5. Stenmark KR, Meyrick B, Galie N, Mooi WJ, McMurtry IF. Animal models of pulmonary arterial hypertension: the hope for etiological discovery and pharmacological cure. Am J Physiol Lung Cell Mol Physiol. 2009;297(6):L1013-32. 
6. Sheikh AQ, Lighthouse JK, Greif DM. Recapitulation of developing artery muscularization in pulmonary hypertension. Cell Rep. 2014;6(5):809-17.

7. Sheikh AQ, Misra A, Rosas IO, Adams RH, Greif DM. Smooth muscle cell progenitors are primed to muscularize in pulmonary hypertension. Sci Transl Med. 2015;7(308):308ra159.

8. Muzumdar MD, Tasic B, Miyamichi K, Li L, Luo L. A global double-fluorescent Cre reporter mouse. Genesis. 2007;45(9):593-605.

9. Wendling O, Bornert JM, Chambon P, Metzger D. Efficient temporally-controlled targeted mutagenesis in smooth muscle cells of the adult mouse. Genesis. 2009;47(1):14-8.

10. Greif DM, Kumar M, Lighthouse JK, Hum J, An A, Ding L, et al. Radial construction of an arterial wall. Dev Cell. 2012;23(3):482-93.

11. Rinkevich Y, Lindau P, Ueno H, Longaker MT, Weissman IL. Germ-layer and lineagerestricted stem/progenitors regenerate the mouse digit tip. Nature. 2011;476(7361):409-13.

12. Ball MK, Waypa GB, Mungai PT, Nielsen JM, Czech L, Dudley VJ, et al. Regulation of hypoxia-induced pulmonary hypertension by vascular smooth muscle hypoxia-inducible factor-1alpha. Am J Respir Crit Care Med. 2014;189(3):314-24.

13. Sheikh AQ, Saddouk FZ, Ntokou A, Mazurek R, Greif DM. Cell autonomous and noncell autonomous regulation of SMC progenitors in pulmonary hypertension. Cell Rep. 2018;23(4):1152-65.

14. Chappell J, Harman JL, Narasimhan VM, Yu H, Foote K, Simons BD, et al. Extensive proliferation of a subset of differentiated, yet plastic, medial vascular smooth muscle cells contributes to neointimal formation in mouse injury and atherosclerosis models. Circ Res. 2016;119(12):1313-23.

15. Jacobsen K, Lund MB, Shim J, Gunnersen S, Fuchtbauer EM, Kjolby M, et al. Diverse cellular architecture of atherosclerotic plaque derives from clonal expansion of a few medial SMCs. JCI Insight. 2017;2(19)

16. Misra A, Feng Z, Chandran RR, Kabir I, Rotllan N, Aryal B, et al. Integrin beta3 regulates clonality and fate of smooth muscle-derived atherosclerotic plaque cells. Nat Commun. 2073;9(1):2018.

Open Access This chapter is licensed under the terms of the Creative Commons Attribution 4.0 International License (http://creativecommons.org/licenses/by/4.0/), which permits use, sharing, adaptation, distribution and reproduction in any medium or format, as long as you give appropriate credit to the original author(s) and the source, provide a link to the Creative Commons license and indicate if changes were made.

The images or other third party material in this chapter are included in the chapter's Creative Commons license, unless indicated otherwise in a credit line to the material. If material is not included in the chapter's Creative Commons license and your intended use is not permitted by statutory regulation or exceeds the permitted use, you will need to obtain permission directly from the copyright holder.

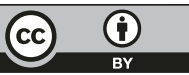

